\title{
Trends in incidence of hypertension in Chinese adults, 1991- 2009: the China Health and Nutrition Survey
}

\author{
Yajun Lianga,b, ${ }^{a}$, Ruijuan Liuc, Shufa Dud, and Chengxuan Qiub, \\ aSchool of Public Health, Jining Medical University, Shandong, China \\ ${ }^{\mathrm{b}}$ Aging Research Center, Department of Neurobiology, Care Sciences and Society, Karolinska \\ Institutet-Stockholm University, Stockholm, Sweden \\ 'Department of Internal Medicine, Jining First People's Hospital, Shandong, China \\ dDepartment of Nutrition, University of North Carolina at Chapel Hill, Chapel Hill, NC, United \\ States
}

\section{Abstract}

Background/objectives-Previous studies have shown an upward trend in the prevalence of hypertension, but data on trend of incidence of hypertension are lacking. We seek to investigate the trends in incidence of hypertension and control of incident hypertension among Chinese adults during 1991-1997 and 2004-2009.

Methods-Within the China Health and Nutrition Survey (1991-2009), we identified five cohorts of adults (age $\geq 18$ years) who were free of hypertension at baseline of each cohort: cohorts 1991-1997 $(\mathrm{n}=4107), 1993-2000(\mathrm{n}=4068), 1997-2004(\mathrm{n}=4141), 2000-2006(\mathrm{n}=4695)$, and 2004-2009 ( $\mathrm{n}=4523$ ). Data on demographics, smoking, alcohol intake, physical activity, body mass index (BMI), and blood pressure were collected through interviews and clinical examination. Hypertension was defined as blood pressure $\geq 140 / 90 \mathrm{mmHg}$ or currently using antihypertensive drugs. Multiple generalized estimation equations and Cox regression models were used to test the trends in blood pressure, incidence of hypertension, use of antihypertensive drugs, and control status of incident hypertension.

Results-After controlling for potential confounders, incidence of hypertension (per 100 personyears) significantly increased from 2.9 in 1991-1997 to 5.3 in 2004-2009 ( $\left.p_{\text {trend }}=0.024\right)$; the linear trend was statistically or marginally significant in the age group of 18-39 years, in women, in rural residents, and in adults with normal BMI. The overall rates of antihypertensive treatment and control of incident hypertension increased significantly from 5.7\% and 1.7\% in 1991-1997 to $19.9 \%$ and $7.6 \%$ in $2004-2009$, respectively $\left(p_{\text {trend }}<0.001\right)$.

(C) 2014 Elsevier Ireland Ltd. All rights reserved.

"Corresponding author at: Aging Research Center, Gävlegatan 16, SE-11330 Stockholm, Sweden. Tel.:+46 86905816; Fax: +46 86905954., yajun.liang@ki.se (Y. Liang) or chengxuan.qiu@ki.se (C. Qiu).

Conflict of interest: There is no conflict of interests in connection with this study.

Publisher's Disclaimer: This is a PDF file of an unedited manuscript that has been accepted for publication. As a service to our customers we are providing this early version of the manuscript. The manuscript will undergo copyediting, typesetting, and review of the resulting proof before it is published in its final citable form. Please note that during the production process errors may be discovered which could affect the content, and all legal disclaimers that apply to the journal pertain. 
Conclusions-The incidence of hypertension has increased in Chinese adults since early 1990s. The treatment and control status of incident hypertension, while improved, remain very poor.

\section{Keywords}

Trends; Incidence; Hypertension; Adults; China

\section{Introduction}

The mortality of coronary heart disease (CHD) and stroke in the United States of America decreased at $\sim 3 \%$ per year between 1970 and 1997 [1]. In England, mortality of CHD had fallen $\sim 6 \%$ every year during 2000-2007 [2]. The decline in incidence and mortality of major cardiovascular diseases (CVDs) among high-income countries is largely attributable to the implementation of various prevention strategies including control of hypertension, high cholesterol, and smoking [1-5]. By contrast, in low- and middle-income countries, although mortality of stroke decreased, the incidence of stroke increased significantly from 1990 to 2010 [6]. In China, the burden of major CVDs (CHD and stroke), as the leading causes of death and disability, has increased over 1990-2010 [7].

Hypertension, as a major modifiable risk factor for CVDs, is fairly common among adults. The World Health Organization (WHO) Study on Global Aging and Adult Health (SAGE) of middle-income countries showed that the prevalence of hypertension varied from $23 \%$ to $52 \%$, with the prevalence among people aged 35 years or older being $39 \%$ in China [8]. Several studies from high-income countries have shown an increase in the prevalence of hypertension since the 1990s [9-11]. Previously, data from the China Health and Nutrition Survey (CHNS) have suggested that the prevalence of hypertension in Chinese adults has increased from $14.5 \%$ in 1991 to $21.4 \%$ in 2009 [12]. However, data on the trend of incidence of hypertension are currently lacking.

In addition, the treatment and control of prevalent hypertension have been improved in many high-income countries. For example, studies from the United States of America showed that the control rate of hypertension had increased from 27.3\% in 1988-1994 to $47.2 \%$ in 2009-2010 [10,13]. By contrast, although the treatment and control rates of hypertension have increased, it remains unacceptably low in middle-income countries including China $[8,12,14]$. The WHO SAGE study of middle-income countries suggested that approximately $73 \%$ of patients with hypertension were untreated and less than $10 \%$ were well controlled during 2007-2010 [8]. Blood pressure (BP) control of patients with hypertension remains a major problem in central and eastern European countries [14]. In China, data from CHNS also show an increase in the control rate of prevalent hypertension from 1991 to 2009, but the rate remained extremely low (6.1\% in 2009) [12].

Previous studies from the CHNS have shown an upward trend in the prevalence of hypertension as well as in the treatment and control rates of prevalent hypertension [12]. While the increase in survival of people with hypertension contributes to the increased prevalence, it is plausible to hypothesize that the increased prevalence is partially owing to the increased incidence of hypertension. In the current analysis, using data from CHNS we seek to test this hypothesis by investigating the secular trends in incidence, treatment, and 
control status of incident hypertension among Chinese adults from 1991-1997 to 20042009.

\section{Methods}

\subsection{Study design and population}

CHNS is a nationwide survey aiming to investigate health and nutritional status in Chinese populations (age $\geq 2$ years) [15]. The study sample for CHNS was drawn from nine provinces (Liaoning, Heilongjiang, Jiangsu, Shandong, Henan, Hubei, Hunan, Guangxi, and Guizhou) through a multistage, randomized, cluster sampling process, as fully described elsewhere [15]. The CHNS survey was conducted in 1989, 1991, 1993, 1997, 2000, 2004, 2006, 2009, and 2011. Because BP was measured only once in the 1989 survey and data on the 2011 survey had not yet been released at the time when this analysis was completed, we included data from the surveys 1991 through 2009. Eligible subjects for this analysis were adults of people aged $\geq 18$ years.

To determine the trend in incidence of hypertension over time, we identified five cohorts that covered five time periods. Each cohort (time period) covered three waves of survey: the first wave was considered as baseline survey, and the two successive waves were considered as follow-up surveys. For each cohort, we excluded (1) subjects who were identified to have hypertension at baseline survey, and (2) subjects who did not participate in either of the two successive follow-up surveys. Therefore, this study was based on the following five cohorts of adults (age $\geq 18$ years) who were free of hypertension at baselines and who completed at least one follow-up survey: cohort 1991-1997 ( $\mathrm{n}=4107)$, cohort 1993-2000 $(\mathrm{n}=4068)$, cohort 1997-2004 $(n=4141)$, cohort 2000-2006 $(n=4695)$, and cohort 2004-2009 $(n=4523)$. Of these, $35.6 \%, 24.0 \%, 19.2 \%, 10.2 \%$, and $10.9 \%$ subjects were included in $1,2,3,4$, and all the 5 cohorts, respectively.

The current study complies with the Declaration of Helsinki. The survey protocols, instruments, and the process for obtaining the informed consent for CHNS participants were approved by the Institutional Review Committees of the University of North Carolina at Chapel Hill, NC, USA, and the China National Institute of Nutrition and Food Safety at the Chinese Center for Disease Control and Prevention, Beijing, China. All participants provided written informed consents prior to the surveys.

\subsection{Data collection and definitions}

Data on demographics (age, sex, education, and living area), BP, weight, height, lifestyles (ever smoking, alcohol intake, and physical activity), and use of medications were collected by trained and certified health professionals through interviews and physical examinations. Ever smoking was defined as a positive answer to the question "have you ever smoked cigarettes or pipe?" Alcohol intake was defined as regular drinking alcoholic beverage $\geq 3$ times a week. Because data on leisure-time physical activities were not collected in the surveys before 1997, physical activity was defined as the combination of occupational activity and home activity, as previously reported [16]. The total metabolic equivalents (METs) of physical activity were calculated as MET-hrs per week. 
After at least a 10-min rest, arterial BP (Korotkoff phase I for systolic and phase V for diastolic pressure) was measured on the right arm in the sitting position using a mercury sphygmomanometer with the cuff maintained at the heart level. BP was measured three times on one visit, and the mean of three readings was used in the analysis. Hypertension was defined as systolic BP (SBP) $\geq 140 \mathrm{~mm} \mathrm{Hg}$, diastolic BP (DBP) $\geq 90 \mathrm{~mm} \mathrm{Hg}$, or current use of antihypertensive medications [17]. Among participants who were identified to have developed incident hypertension, control of hypertension was defined as SBP $<140 \mathrm{~mm} \mathrm{Hg}$ and DBP $<90 \mathrm{~mm} \mathrm{Hg}$ after receiving antihypertensive medications [17].

Height was measured to the nearest $0.1 \mathrm{~cm}$ without shoes using a portable stadiometer, and weight was measured to the nearest $0.1 \mathrm{~kg}$ with lightweight clothing on a calibrated digital floor scale. Body mass index (BMI) was calculated as weight in kilograms divided by height in meters squared. Overweight or obesity was defined as BMI $\geq 25 \mathrm{~kg} / \mathrm{m}^{2}$.

\subsection{Statistical analysis}

Characteristics of participants in each cohort were presented with mean (standard deviation, SD) for normal distributed continuous variables and with frequency $(\%)$ for categorical variables. We described the trends in mean baseline BP of participants in each cohort and in the rates of treatment and control of hypertension according to age groups (18-39, 40-59, and $\geq 60$ years), sex, region (urban and rural areas), or BMI status (normal weight and overweight or obesity) by plotting linear graphs. The statistical significance of trends in mean BP and in the treatment and control of incident hypertension were assessed by multiple generalized estimation equations (GEE) regression models to address the correlation of repeated measures within individuals. For assessing the trend in mean BP, an identity link and a normal distribution were used in the GEE model, in which SBP or DBP was used as the independent variable. For assessing the trend in the treatment and control rates of incident hypertension, a logit link and a binomial distribution were used in the model, with the treatment or control of hypertension being the dependent variable.

We calculated the incidence of hypertension according to person-years of follow-up (per 100 person-years). For participants who did not develop hypertension at the last contact of follow-up, the follow-up time was calculated from the date of baseline survey to the date of last contact. For those who developed hypertension, date of onset of hypertension was assumed to be in the midpoint between the two surveys because of the insidious onset of hypertension, and thus, the follow-up time was estimated as the full time during which subjects remained free of hypertension plus half of the follow-up time during which hypertension developed. Cox model was employed to assess the trend of incidence of hypertension across five time periods with follow-up time being the time scale.

We controlled for demographic and lifestyle factors to assess their influence on the trend of incidence, treatment, and control rates of hypertension. Because each factor had limited effect on the trend, we reported the results ( $p$ for trend) from the models that controlled for age, sex, region, ever smoking, alcohol intake, physical activity, and BMI at baseline. 
IBM SPSS Statistics 19 for Windows (IBM SPSS Inc., Chicago, Illinois, USA) was used for all analyses. Statistical significance was set at $p \$ \$ 0.05$, and statistically marginal significance at $0.05<p<0.10$.

\section{Results}

Table 1 presents the baseline characteristics of participants by five cohorts. The mean age of participants increased from 40.6 (SD, 13.3) years in cohort 1991-1997 to 46.6 (13.0) years in cohort 2004-2009 ( $\left.p_{\text {trend }}<0.001\right)$. The proportions of people aged 760 years increased over time ( $\left.p_{\text {trend }}<0.001\right)$, while the proportion of rural residents decreased significantly across the five cohorts $\left(p_{\text {trend }}<0.001\right)$. The proportion of female participants slightly increased over time ( $\left.p_{\text {trend }}=0.05\right)$. After controlling for age, sex, and region, mean BMI and prevalence of overweight or obesity significantly increased across five time periods, while the prevalence of ever smoking decreased significantly from cohort 1991-1997 to cohort 2004-2009 (all $p_{\text {trend }}<0.001$ ). There appeared to be a J-shaped trend in physical activity across cohorts $\left(p_{\text {trend }}<0.001\right)$. There was no significant linear trend in the prevalence of alcohol intake over time $\left(p_{\text {trend }}=0.411\right)$.

Fig. 1 shows the trends in mean baseline SBP and DBP from cohort 1991-1997 to cohort 2004-2009 by age, sex, region, and BMI status. For each cohort, mean levels of baseline SBP and DBP were significantly higher in older than in young adults, in men than in women, and in overweight or obese adults than in those with normal BMI $(p<0.001)$, while there was no significant difference in mean BP between urban and rural residents $(p>0.05)$. Controlling for age, sex, region, ever smoking, alcohol intake, physical activity, and BMI, mean baseline SBP and DBP increased significantly from cohort 1991-1997 to cohort 2004-2009 ( $\left.p_{\text {trend }}<0.001\right)$; the linear trends were statistically significant in all subgroups by age, sex, region, and status of overweight or obesity ( $\left.p_{\text {trend }}<0.05\right)$ (Fig. 1).

Table 2 presents the trends in incidence of hypertension over time from 1991-1997 to 20042009 by age, sex, region, and BMI status. Overall, the incidence (per 100 person-years) of hypertension increased from 2.9 in 1991-1997 to 5.3 in 2004-2009 ( $\left.p_{\text {trend }}=0.024\right)$. After controlling for age, sex, region, ever smoking, alcohol intake, physical activity, and BMI, the linear trends of increasing incidence of hypertension were statistically significant in women $\left(p_{\text {trend }}=0.050\right)$, in rural residents $\left(p_{\text {trend }}=0.009\right)$, and in adults with normal BMI $\left(p_{\text {trend }}<0.001\right)$ and marginally significant in the age group of $18-39$ years $\left(p_{\text {trend }}=0.060\right)$. In each of the five cohorts, the incidence of hypertension was significantly higher in older age groups than in younger ones, in men than in women, and in overweight or obese adults than in those with normal BMI $(p<0.01)$, while there was no significant difference in the incidence of hypertension between urban and rural residents $(p>0.05)$.

The self-reported antihypertensive treatment and control status of incident hypertension in five cohorts by age, sex, region, and BMI status are shown in Fig. 2. First, among people with incident hypertension, the overall rate of self-reported antihypertensive treatment increased from $5.7 \%$ in 1997 to $19.9 \%$ in 2009 ( $p_{\text {trend }}<0.001$ ). The rate of antihypertensive treatment increased significantly over time in all subgroups $\left(p_{\text {trend }}<0.01\right)$, except for young adults aged 18-39 years $\left(p_{\text {trend }}=0.098\right)$. Second, the rate of antihypertensive treatment was 
higher in older than in young adults, and in women than in men from 2004 to 2009, whereas the rate of treatment was higher in urban than in rural residents, and in overweight or obese people than in those with normal weight from 1997 to 2009.

Among people with incident hypertension, the overall control rate of incident hypertension increased significantly from $1.7 \%$ in 1997 to $7.6 \%$ in 2009 ( $\left.p_{\text {trend }}<0.001\right)$. The control rate increased significantly over time in all subgroups $\left(p_{\text {trend }}<0.01\right)$, except for people aged 18 39 years $\left(p_{\text {trend }}=0.226\right)$. The control rate was higher in older than in young adults, and in women than in men in 2006 and 2009, and in urban than in rural residents across all cohorts, whereas overweight or obese adults had a significantly or marginally higher control rate than those with normal weight only in $2004(p=0.039)$ and $2009(p=0.051)$.

\section{Discussion}

This population-based study from CHNS suggested that, among Chinese adults, the incidence of hypertension increased over time from 1991-1997 to 2004-2009, especially in young adults, women, rural residents, and those with normal weight. The incidence of hypertension was higher in older than in young adults, in men than in women, and in overweight or obese adults than in those with normal weight. The rates of self-reported antihypertensive treatment and control among Chinese adults with incident hypertension, although increased over time, remained very low.

We previously reported that prevalence of hypertension in Chinese adults increased during 1991 and 2009 [12]. The current analysis suggested that the increased trend in prevalence of hypertension is at least partly owing to the increased incidence of hypertension because the trend is present independent of major influential factors of BP such as age, BMI, physical activity, and living region. In addition, our data showed that the baseline level of BP in people free of hypertension increased over time, which may contribute to the increased trend in incidence of hypertension. The increased trend in SBP over time among Chinese adults without hypertension is in accordance with the report from the United States national survey of adults who are free of hypertension [10]. Indeed, the reports from the United States survey and the WHO Multinational Monitoring Trends and Determinants in CVD Project suggested a trend of decrease in SBP and DBP from the 1980s to the 2000s in the whole population $[10,18]$, which is largely owing to improvement in therapeutic control of high BP among the general populations.

The incidence of hypertension varied with age, sex, and living area of participants. As expected, BP level and incidence of hypertension were higher in older people than in young adults. However, the increased trend in incidence of hypertension was evident mainly in young adults. This suggests that young Chinese adults increasingly are affected by high BP, which has significant implications for public health. The incidence of hypertension increased over time in women but not in men, although the incidence was higher in men than in women, which are in agreement with the prevalence data [12,19,20]. Moreover, previous studies have indeed revealed a higher prevalence of hypertension in urban than rural residents $[19,20]$, but the rural-urban difference in prevalence of hypertension tended to diminish in the last decade [12]. We found that the incidence of hypertension was 
generally comparable between rural and urban residents, but the increased trend in incidence of hypertension was evident only in those living in rural area. This may partly reflect the fact that the considerable urban-rural gap in lifestyles and socioeconomic status existing before the 1990s in China had gradually diminished in the last decade or so; rural areas have acquired certain characteristics of urban environments as a result of quick industrialization and urbanization, which may promote development of chronic diseases such as hypertension $[21,22]$.

Changes in lifestyles and BP-related factors may contribute to the increase in incidence of hypertension. First, overweight or obesity is strongly associated with hypertension [19,2325]. Data from CHNS showed that during 1993-2009, there was an upward trend in prevalence of obesity in Chinese adults $[23,26]$. However, this cannot explain the increased trend in incidence of hypertension because the trend was present independent of overweight or obesity, and was evident mainly among people with normal BMI. Second, although physical activity (assessed as the combination of occupational and home physical activities) tended to increase from the survey 1997 onward, the meta-analysis revealed no convincing evidence for the association between occupational physical activity and risk of hypertension [27]. Third, urbanization, which has accelerated in China during the study period, might contribute to the increased trend in incidence of hypertension, because the urbanization and related changes in living conditions are linked to certain risk factors for hypertension, such as increasing intake in dietary fats, stressful life, and sedentary lifestyle among Chinese residents $[16,22]$. Fourth, the high intake of sodium, along with the increasing prevalence of obesity or overweight and heavy alcohol consumption, might partially explain the increasing incidence of hypertension among Chinese adults [28,29]. Finally, along with rapid urbanization and industrialization in China, the increasing outdoor air pollution might also partly contribute to the increased trend in the incidence of hypertension [22,30].

In the last decade, several studies have consistently shown an increasing trend in the treatment and control rate of prevalent hypertension $[10,12,13]$. We found a trend towards an overall improvement in the treatment and control rate of incident hypertension over time. However, among persons who were identified to have developed incident hypertension, the proportions of those receiving antihypertensive treatment and of achieving the goal of BP control were very low compared to studies of prevalent hypertension [10-12]. This may not be surprising given that incident hypertension is by definition the newly developed hypertension, and a considerable proportion of people with newly developed hypertension over the 5-7 year period are likely to be unaware of the disease. Nevertheless, the rates of treatment and control of incident hypertension were higher in older people than younger adults, in women than men, in urban residents than rural people, and in obese adults than in those with normal weight, which were consistent with the results of treatment and control of prevalent hypertension from CHNS [12]. These results imply that closely monitoring BP among Chinese adults, especially young adults, may help avoid any delay of antihypertensive treatment.

This study is based on a large-scale nationwide sample in China that covers a wide range of demographics, socioeconomic status, and nutritional and health conditions. Moreover, a standardized approach in BP measurement has been used consistently across the whole 
period that comprises multiple assessments over time. However, this study also has limitations. Firstly, given the tremendous diversity of Chinese population across regions, the size and representativeness of the study sample for each cohort remained limited. Secondly, BP was measured at only one occasion without follow-up visits, which might have overestimated the incidence of hypertension [10]. Finally, data on use of antihypertensive medications were self-reported, not verified independently.

In conclusion, the incidence of hypertension has increased steadily over the last two decades among Chinese adults, especially in young adults, women, rural residents, and those with normal BMI. A considerable proportion ( $>80 \%)$ of people with incident hypertension are not timely treated, and even fewer $(<10 \%)$ achieve the goal of BP control. Given that CVDs are the leading cause of deaths and disability-adjusted life-years in China, and that hypertension, especially when leaving untreated or uncontrolled, is the strongest single risk factor for CVDs, the government-dominated nationwide campaign to improve the management of hypertension is indicated to reduce the tremendous burden of CVDs in China.

\section{Acknowledgments}

This research uses data from the China Health and Nutrition Survey (CHNS). We thank the National Institute of Nutrition and Food Safety, China Center for Disease Control and Prevention; the Carolina Population Center, University of North Carolina at Chapel Hill; the National Institutes of Health (NIH; R01-HD30880, DK056350, and R01-HD38700); and the Fogarty International Center, NIH, for financial support for the CHNS data collection and analysis files since 1989. We thank those parties, the China-Japan Friendship Hospital, and the Ministry of Health for support for CHNS 2009 and future surveys. Dr Qiu was supported by grants from the Swedish Research Council and Karolinska Institutet, Stockholm, Sweden.

\section{References}

1. Cooper R, Cutler J, Desvigne-Nickens P, et al. Trends and disparities in coronary heart disease, stroke, and other cardiovascular diseases in the United States: findings of the national conference on cardiovascular disease prevention. Circulation. 2000; 102:3137-47. [PubMed: 11120707]

2. Bajekal M, Scholes S, Love H, et al. Analysing recent socioeconomic trends in coronary heart disease mortality in England, 2000-2007: a population modelling study. PLoS Med. 2012; 9:e1001237. [PubMed: 22719232]

3. Redon J, Olsen MH, Cooper RS, et al. Stroke mortality and trends from 1990 to 2006 in 39 countries from Europe and Central Asia: implications for control of high blood pressure. Eur Heart J. 2011; 32:1424-31. [PubMed: 21487117]

4. Rosengren A, Giang KW, Lappas G, Jern C, Torén K, Björck L. Twenty-four-year trends in the incidence of ischemic stroke in Sweden from 1987 to 2010. Stroke. 2013; 44:2388-93. [PubMed: 23839506]

5. Di Cesare M, Bennett JE, Best N, Stevens GA, Danaei G, Ezzati M. The contributions of risk factor trends to cardiometabolic mortality decline in 26 industrialized countries. Int J Epidemiol. 2013; 42:838-48. [PubMed: 23744994]

6. Feigin VL, Forouzanfar MH, Krishnamurthi R, et al. Global and regional burden of stroke during 1990-2010: findings from the Global Burden of Disease Study 2010. Lancet. 2014; 383:245-254. [PubMed: 24449944]

7. Yang G, Wang Y, Zeng Y, et al. Rapid health transition in China, 1990-2010: findings from the Global Burden of Disease Study 2010. Lancet. 2013; 381:1987-2015. [PubMed: 23746901]

8. Basu S, Millett C. Social epidemiology of hypertension in middle-income countries: determinants of prevalence, diagnosis, treatment, and control in the WHO SAGE Study. Hypertension. 2013; 62:1826. [PubMed: 23670299] 
9. Hotchkiss JW, Davies C, Gray L, Bromley C, Capewell S, Leyland AH. Trends in adult cardiovascular disease risk factors and their socio-economic patterning in the Scottish population 1995-2008: cross-sectional surveys. BMJ Open. 2011; 1:e000176.

10. Egan BM, Zhao Y, Axon RN. US trends in prevalence, awareness, treatment, and control of hypertension, 1988-2008. JAMA. 2010; 303:2043-50. [PubMed: 20501926]

11. Hajjar I, Kotchen JM, Kotchen TA. Hypertension: trends in prevalence, incidence, and control. Annu Rev Public Health. 2006; 27:465-90. [PubMed: 16533126]

12. Xi B, Liang Y, Reilly KH, Wang Q, Hu Y, Tang W. Trends in prevalence, awareness, treatment, and control of hypertension among Chinese adults 1991-2009. Int J Cardiol. 2012; 158:326-29. [PubMed: 22626836]

13. Gu Q, Burt VL, Dillon CF, Yoon S. Trends in antihypertensive medication use and blood pressure control among United States adults with hypertension: the National Health and Nutrition Examination Survey, 2001 to 2010. Circulation. 2012; 126:2105-14. [PubMed: 23091084]

14. Grassi G, Cifkova R, Laurent S, et al. Blood pressure control and cardiovascular risk profile in hypertensive patients from central and eastern European countries: results of the BP-CARE study. Eur Heart J. 2011; 32:218-25. [PubMed: 21047877]

15. Popkin BM, Du S, Zhai F, Zhang B. Cohort Profile: The China Health and Nutrition Survey-monitoring and understanding socio-economic and health change in China, 1989-2011. Int J Epidemiol. 2010; 39:1435-40. [PubMed: 19887509]

16. Ng SW, Norton EC, Popkin BM. Why have physical activity levels declined among Chinese adults? Findings from the 1991-2006 China Health and Nutrition Surveys. Soc Sci Med. 2009; 68:1305-14. [PubMed: 19232811]

17. Chobanian AV, Bakris GL, Black HR, et al. The Seventh Report of the Joint National Committee on Prevention, Detection, Evaluation, and Treatment of High Blood Pressure: the JNC 7 report. JAMA. 2003; 289:2560-72. [PubMed: 12748199]

18. Tunstall-Pedoe H, Connaghan J, Woodward M, Tolonen H, Kuulasmaa K. Pattern of declining blood pressure across replicate population surveys of the WHO MONICA project, mid-1980s to mid-1990s, and the role of medication. BMJ. 2006; 332:629-35. [PubMed: 16531419]

19. Wu Y, Huxley R, Li L, et al. Prevalence, awareness, treatment, and control of hypertension in China: data from the China National Nutrition and Health Survey 2002. Circulation. 2008; 118:2679-86. [PubMed: 19106390]

20. Xu B, Xu Z, Xu X, Cai Q, Xu Y. Prevalence, awareness, treatment, and control of hypertension among residents in Guangdong Province, China, 2004 to 2007. Circ Cardiovasc Qual Outcomes. 2013; 6:217-22. [PubMed: 23481521]

21. Du S, Mroz TA, Zhai F, Popkin BM. Rapid income growth adversely affects diet quality in China-particularly for the poor! Soc Sci Med. 2004; 59:1505-15. [PubMed: 15246178]

22. Gong P, Liang S, Carlton EJ, et al. Urbanisation and health in China. Lancet. 2012; 379:843-52. [PubMed: 22386037]

23. Du T, Sun X, Yin P, Huo R, Ni C, Yu X. Increasing trends in central obesity among Chinese adults with normal body mass index, 1993-2009. BMC Public Health. 2013; 13:327. [PubMed: 23575244]

24. Nguyen NT, Magno CP, Lane KT, Hinojosa MW, Lane JS. Association of hypertension, diabetes, dyslipidemia, and metabolic syndrome with obesity: findings from the National Health and Nutrition Examination Survey, 1999 to 2004. J Am Coll Surg. 2008; 207:928-34. [PubMed: 19183541]

25. Deng WW, Wang J, Liu MM, et al. Body mass index compared with abdominal obesity indicators in relation to prehypertension and hypertension in adults: the CHPSNE study. Am J Hypertens. 2013; 26:58-67. [PubMed: 23382328]

26. Xi B, Liang Y, He T, et al. Secular trends in the prevalence of general and abdominal obesity among Chinese adults, 1993-2009. Obes Rev. 2011; 13:287-96. [PubMed: 22034908]

27. Huai P, Xun H, Reilly KH, et al. Physical activity and risk of hypertension: a meta-analysis of prospective cohort studies. Hypertension. 2013; 62:1021-6. 
28. Stamler R, Liu LS, Nichols R, et al. Blood pressure and life style in the People's Republic of China: three samples in the INTERSALT Study. J Hum Hypertens. 1993; 7:429-35. [PubMed: 8263882]

29. Batis C, Gordon-Larsen P, Cole SR, Du S, Zhang B, Popkin B. Sodium intake from various time frames and incident hypertension among Chinese adults. Epidemiology. 2013; 24:410-18. [PubMed: 23466527]

30. Dong GH, Qian ZM, Xaverius PK, et al. Association between long-term air pollution and increased blood pressure and hypertension in China. Hypertension. 2013; 61:578-84. [PubMed: 23357184] 


\section{Highlights}

- The incidence of hypertension has increased in Chinese adults since early 1990 s.

- The trend is obvious in young, women, rural residents, and those with normal BMI.

- The treatment and control status of incident hypertension remain very poor. 

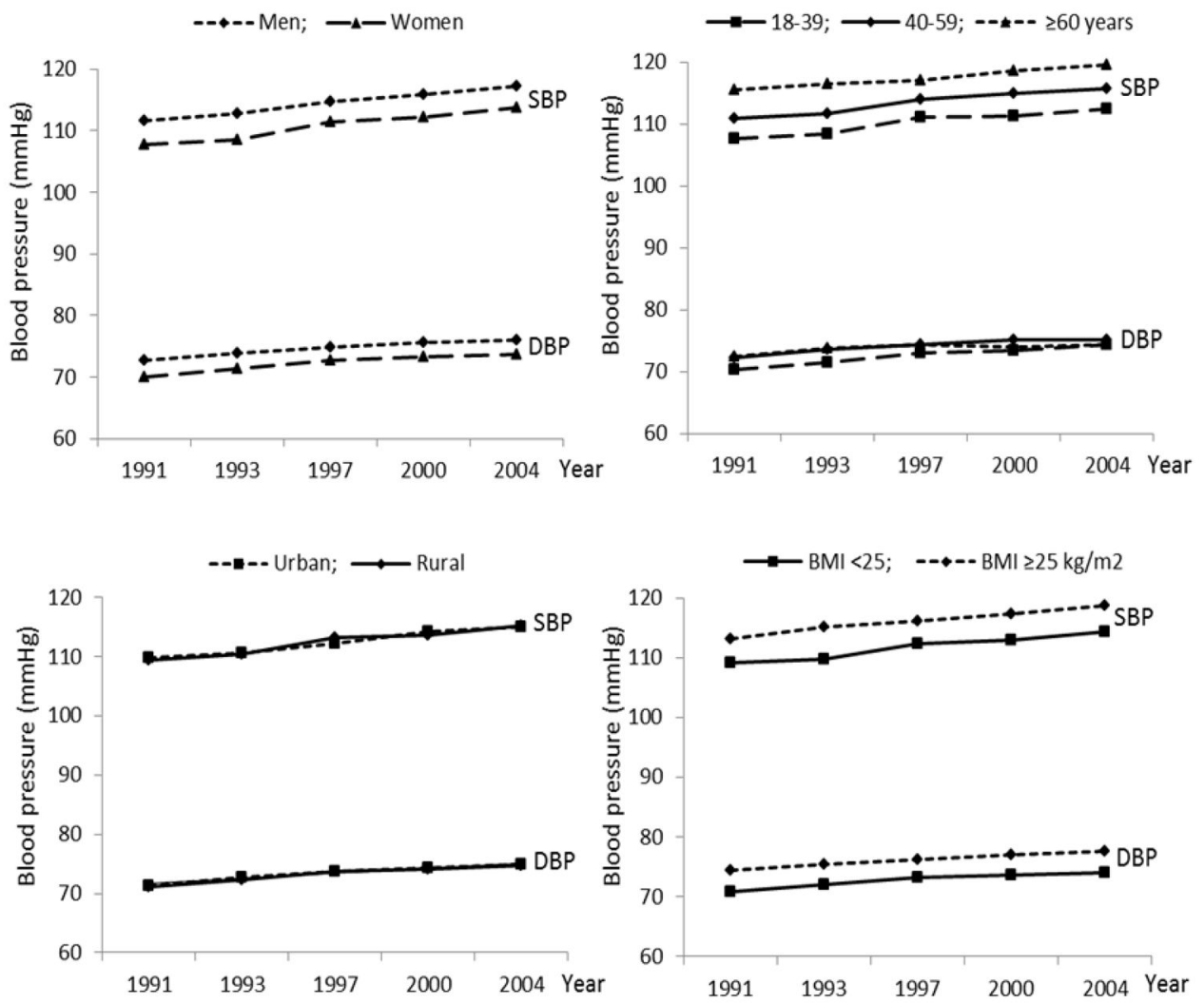

Fig. 1.

Mean systolic blood pressure (SBP) and diastolic blood pressure (DBP) at baseline of five cohorts of adults who were free of hypertension by sex, age, region, and body mass index (BMI)

Controlling for age, sex, region, ever smoking, alcohol intake, physical activity, and body mass index, $p_{\text {trend }}<0.05$ for the mean SBP and DBP from 1991 to 2004 in all subgroups. 

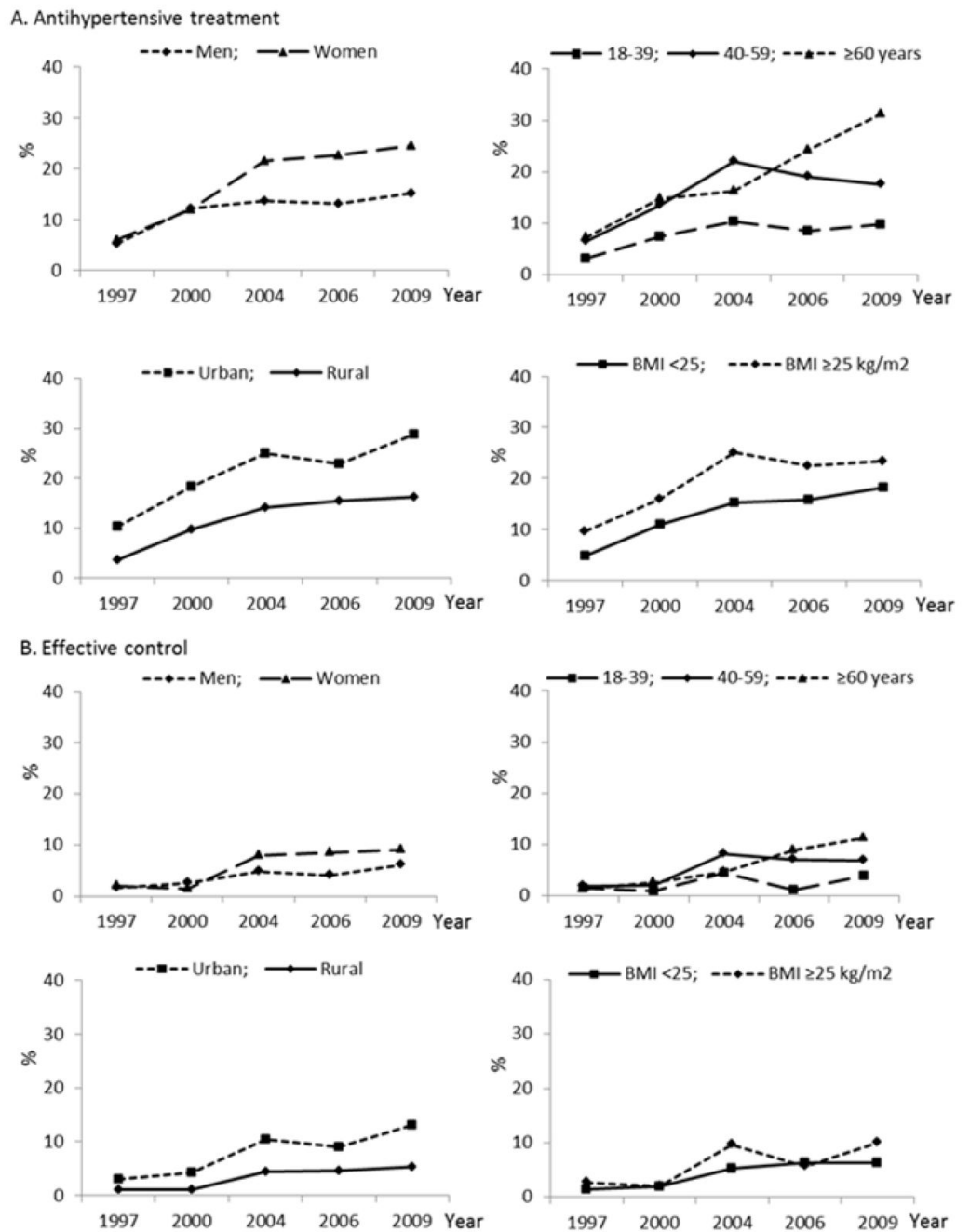

Fig. 2.

The proportion of self-reported antihypertensive treatment (A) and control (B) of incident hypertension in five cohorts by sex, age, region, and body mass index (BMI) Controlling for age, sex, region, ever smoking, alcohol intake, physical activity, and body mass index at baseline, $p_{\text {trend }}<0.01$ for the rates of antihypertensive treatment and effective control of incident hypertension from 1997 to 2009 in all subgroups, except for people aged $18-39$ years $\left(p_{\text {trend }}=0.098\right.$ for rate of antihypertensive treatment; $p_{\text {trend }}=0.226$ for control rate of incident hypertension). 


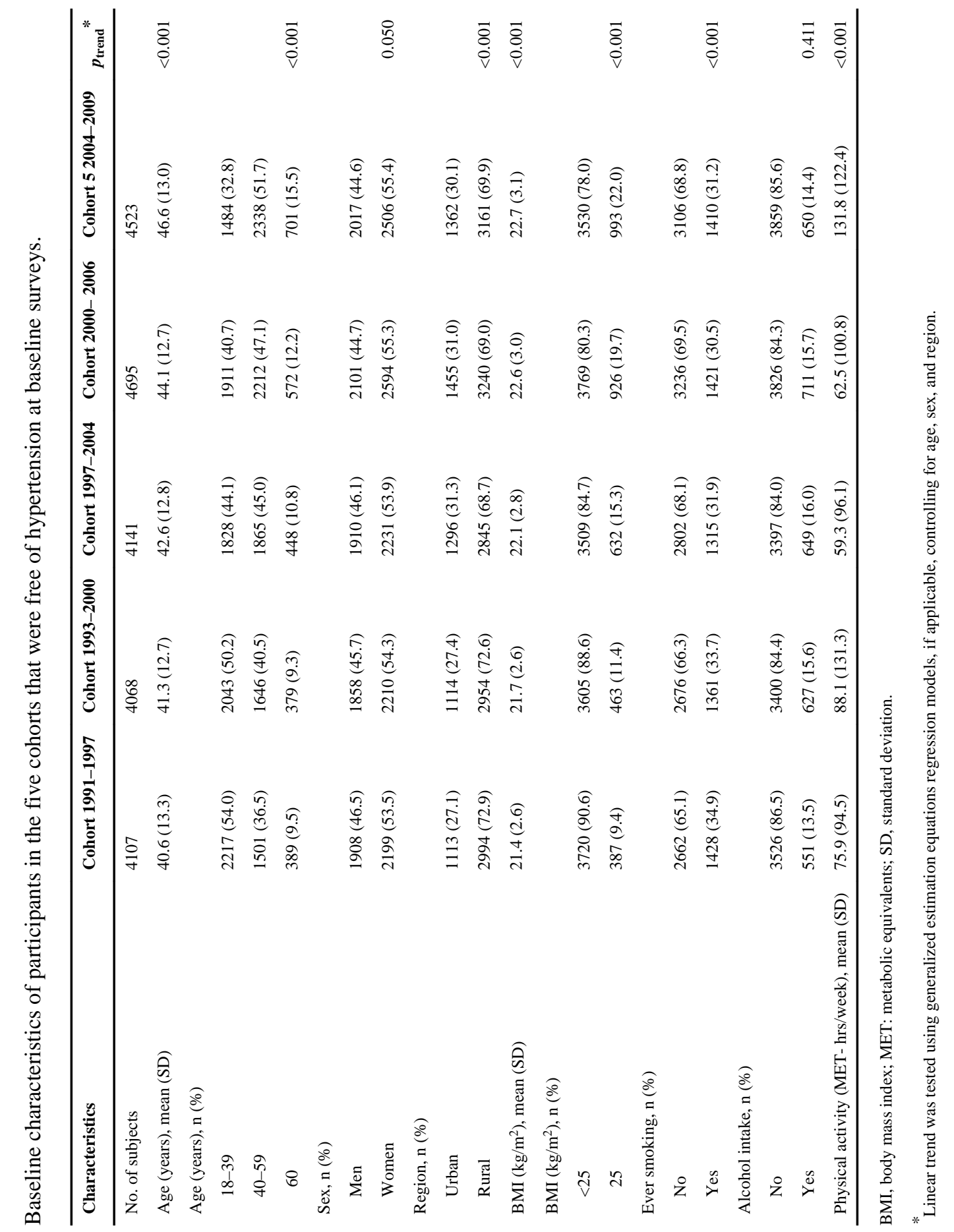


